$$
\text { conif }-8402 \times 0 \cdots-8
$$

CONF $-840230--8$

DES 4014110

\title{
RESULTS ON NEUTRINO-ELECTRON ELASTIC SCATTERING AT AGS ENERGIES
}

Authors: L. A. Ahrens, S. H. Aronson, P. L. Connully, B. G. Gibbard, Y. Maeda, M. J. Murtagh, S. J. Murtagh, S. Terada, D. H. White, Brookhaven Nalional Laboratory; J. L.Callas, D. Cutts, J. Hoftun, R. E. Lanou, T. Shinkawa, Brown University; K. Amako, S. Kabe, (ㅌEK) Japan National Laboratory for High Energy Physics; Y. Nagashima. Y. Suzuki, and S. Tatsumi, Osaka University; K. Abe, E. W. Beier, D. C. Doughty, L. S. Durkin, S. M. Heagy, M. Hurley, A. K. Mann, H. H. Williams, T. York, University of Pennsylyania; D. Hedin. M. D. Marx, and E. Stern, SUNY (Stony Brook).

\author{
Presented by R. E. Lanou \\ Department of Physics \\ Brown University \\ Providence, Rhode Island 02912 USA
}

\begin{abstract}
In an experiment designed to study elastic scattering of $v_{\mu}$ (and $\bar{v}_{u}$ ) from electrons and protons via the weak neutral current, we have recently completed several_extensive data taking runs. Results for the cross section of $v_{1}+e^{-}+v_{+}+e^{-}$are presented based upon the first third of the available sample. 'Preliminary analysis of $v_{\mu}+s_{-}^{-} \bar{v}_{\mu}+e^{-}$is in progress and evidence for the signal is presentel.
\end{abstract}

(Paper given at XIX Rencontre de loriond, 26 February - 5 Marcì 1984 (La Plagne, France))

\section{DISCLAIMER}

This report was prepared as an account of work sponsored by an agency of the United States Government. Neither the United States Government nor any agency thereof, nor any of their employees, makes any warranty, express or implied, or assumes any legal liability or responsibility for the accuracy, completeness, or usefulness of any information, apparatus, profuct, or process disclosed, or represents that its use would not infringe privately owned rights. Peference herein to any specific commercial product, process, or service by trade name, trademark, manufacturer, or otherwise does not necessarily constitute or imply its endorsement, recommendation, or favoring by the United States Government or any agency thereof. The views and ipinions of authors expressed herein do not necessarily state or reflect those of the United States Government or any agency thereof.

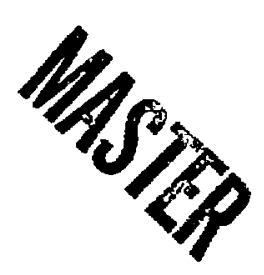


The purely leptonic, weak neutral current reactions:

$$
\begin{aligned}
& v_{\mu}+e^{-} \rightarrow v_{\mu}+e^{-} \\
& \bar{v}_{\mu}+e^{-}+\bar{v}_{\mu}+e^{-}
\end{aligned}
$$

are prescribed completely by the $S U(2)_{L} \times D(1)$ "Standard Mode1". The cross sections are smal1, being the order of $10^{-42} \mathrm{~cm}^{2}$ at $E=1 \mathrm{GeV}$, and are given by

$$
\sigma\left(\frac{v}{v}\right)=\frac{G_{F}^{2} m_{e}}{2 \pi} E_{v} \rho^{2}\left[\left(g_{v}^{e} \pm g_{A}^{e}\right)^{2}+\frac{1}{3}\left(g_{v}^{e} \pm g_{A}^{e}\right)^{2}\right]
$$

Where $g_{V}$ and $g_{A}$ are the vector and axial vector coupling of the electron to the neutral, intermeciate vector boson $\left(Z^{\circ}\right)$, p can be taken as the neutrino's coupling to the $z^{0}$ and $G_{F}$ is the Fermi constant. In the 'Standard Model'', the expectation is that $\mathrm{g}_{A}{ }^{\mathrm{e}}=-1 / 2, \mathrm{~g}_{\mathrm{V}}{ }^{\mathrm{e}}=-1 / 2+$ $2 \sin ^{2} \theta_{w}$, and $\rho=1$ if the Higgs' boson is in an iso-doublet.

A precision measurement of the parameter, $\sin ^{2} \theta$, in purely leptonic reactions is important because it is relatively free from the uncertainties due to QCD radiative corrections. ${ }^{1)}$

The precision achievable in experiments utilizing reaction (1) and (2) is emphasized by forming the ratio of their cross sections written explicitly in terms of $\sin ^{2} \theta_{w}$ :

$$
R=\frac{\sigma\left(\nu_{\mu}-e^{-}\right)}{\sigma\left(\bar{\nu}_{\mu}-e^{-}\right)}=3\left\{\frac{\left(1-4 \sin ^{2} \theta_{w}+\frac{16}{3} \sin ^{4} \theta_{w}\right)}{\left(1-4 \sin ^{2} \theta_{w}+16 \sin ^{4} \theta_{w}\right)}\right\}
$$

The error in $\sin ^{2} \theta_{w}$ depends upon the value of $\sin ^{2} \theta_{w}$ and is approximately one eighth the error in $R$ when $\sin ^{2} \theta_{w}=1 / 4--$ a condition apparently nearly satisfied in nature.

The aim in the experiment under discussion here is to measure the $\sin ^{2} \theta_{w}$ as precisely as possible at low neutrino energy ( $\left.1 \mathrm{GeV}\right)$. The fundamental limit to the precision is expected to arise from systematic uncertainties principally due to relative flur normalization.

The experiment is being performed at the Alternating Gradient Synchrotron of the Brookhaven National Laboratory. The detector consists of an active material (liquid scintillator) which also serves as a calorimeter $\left(\Delta E / E=0.15 /(E)^{1 / 2}\right)$ and to provide fast timing . Figure 1 and Table I contain the details of the detector paxameters. The 
BROOKHAVEN NEUTRINO DETECTOR

LOCATION

Brookhaven Nationa 1 Laboratory, Upton, NY 11973 USA

INCIDENT BEAM Neutrino, Horn Focussed from $28 \mathrm{GeV}$ Protons $110 \mathrm{~m}$ from proton target

ASSEMBLY

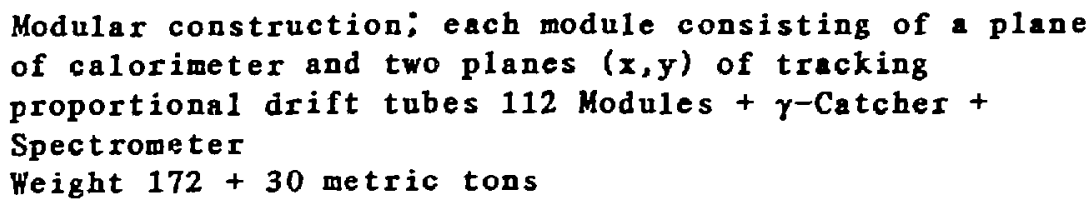

MODULE PROPERTIES

\section{CALORIMETER (LIQUID SCINTILLATOR)}

Active Area $4.22 \times 4.09 \mathrm{~m}^{2}$ Thickness $7.9 \mathrm{~cm}$

Weight (Liquid and Acrylic) 1.35 metric tons

16 cells/module 2 Amperex 2212A phototubes/cel1

1 Pulse Height Measurement/2 Time Measurements per Tube Readout

PROPORTIONAL DRIFT TUBES (PDT)

Active Area $4.2 \times 4.2 \mathrm{~m}^{2}$

Thickness ( $x$ and $y$ ) $7.6 \mathrm{~cm} 54 x$ wires $54 \mathrm{y}$ wires

1 Pulse Height Measurement/2 Time Measurements per Wire Readout

GAMMA CATCHER 10 standard calorimeter modules with 2 radiation

length of lead between each module

30 metric tons target mass.

MUON SPECTROMETER

$$
\begin{aligned}
& 2 \mathrm{~m} \times 2 \mathrm{~m} \text { Aperture Muon Spectrometer } \\
& \langle(\mathrm{Bd} 1\rangle=70 \mathrm{MeV} / \mathrm{c} \\
& (\Delta \mathrm{p} / \mathrm{p})^{2}=\left(.10^{2}+(.067 \mathrm{p})\right)^{2} \mathrm{p} \text { in } \mathrm{GeV} / \mathrm{c}
\end{aligned}
$$

TABLE II

\begin{tabular}{|c|c|c|c|}
\hline Run No. & v & $\overline{\bar{y}}$ & Fid. Vol. \\
\hline I & $6.3 \times 10^{18}$ & $5 \times 10^{17}$ & $1 / 2$ \\
\hline II & $8.8 \times 10^{18}$ & $9.1 \times 10^{18}$ & 1 \\
\hline III & $1.4 \times 10^{19}$ & $2.7 \times 10^{19}$ & 1 \\
\hline
\end{tabular}

Data Runs Accumulated

Protons on Target 
construction is modular with each calorimeter plane matcred with a proportional drift tube plene pair $(X, Y)$ which provides precision measurements of angle $\left(\Delta \theta=0.022 \mathrm{rad} /(E)^{1 / 2}\right.$ for electrons ) as well as additional pulse height and timing information. A magnetic spectrometer (air gap) follows the detector and is used to aid in establishing the properties of the neutrino (anti-neutrino) flux.

The detector is operated in a triggez-less mode via a pre-beam initiated $10 \mathrm{ksec} g$ ate during which the beam is extracted in 12 R.F.-buckets of 30 nsec duration separated by 224 nsec. The beam pulse repetition rate is approrimately 40 per minute. The average bean energy is approximately $1.5 \mathrm{GeV}$ for $\nu_{\mu}$ and $1.3 \mathrm{GeV}$ for $\bar{\nu}_{\mu}$. This bean structure permits continuous monitoring of possible out-of-time backgrounds $\left(<1 x_{0}\right)$ and initial event selection by time-clustering of hits.

The kinematics of the reactons (1) and (2) provide a distinctive signal. In particular, the relation $\mathrm{E}_{\mathrm{e}} \theta_{\mathrm{e}}{ }^{2}=2 \mathrm{~m}_{\mathrm{e}}\left(1-\mathrm{E}_{\mathrm{e}} / \mathrm{E}\right)$ restricts events to very small laboratory angles.

Here $\theta_{e}$ and $E_{e}$ are the laboratory angle and energy, respectively, of the recoil electron. From this relation it can be seen that all of the signal is contained at $\mathrm{E}_{\mathrm{e}} \theta_{\mathrm{e}}{ }^{2}<2 \mathrm{~m}_{\mathrm{e}}$ exclusive of resolution. At AGS energies the mean energy of the recoil electron is roughly $500 \mathrm{MeV}$ thus the corresponding r.m.s. angles are less than 0.045 radians. The signal is consequently very strongly forward peaked.

Details of the analysis procedure can be found elsewhere: ${ }^{2}$ ) however a brief discussion is given here.

The event sample to be studied is initially selected from the raw data tapes by loose software filters designed to select forward-going $\left(<20^{\circ}\right.$ ) events which do not contain muons or extra hadrons. The resulting sample is then cut to contain only events with shower angles less than $180 \mathrm{mr}$; this sample contains more than 90\% of the reaction (1) and (2) events.

The prinicpal backgrounds arise from the quasi-elastic reactions involving the small contamination of $\nu_{e}$ and $\bar{y}_{e}(<0.5 \%)$ in the respective beams and from the neutral current production of $\pi^{0}$ mesons in which some fragments in the final state are not detected and only an electromagnetic shower remains. However, the angular and energy correlations of the background showers are quite different from those of the signal. At AGS energies $\theta^{2}$ distributions are relatively flat and the electron energies from the ${ }^{\prime}$ e reactions are large. To aid in suppression of these 
backgrounds cuts are made on $\theta_{e}^{2}\left(0-0.01 \mathrm{rad}^{2}\right)$ and $E_{e}(210-2100 \mathrm{MeV})$ after an initial selection has been made to separate electromagnetic showers predominantly photon initiated.

This selection is carried out by using the ionization energy deposits in the early part of the showar where photon-initiated showers tend to have more than minimum ionization deposit. The algorithm to achieve this uses the pulse height information from the first calorimeter plane after the vertex and the average of minimum (of $X$ and $Y$ ) pulse beights for the first two proportional drift tube pairs. The acceptable pulse height levels are set by comparison to several reference samples: beam muons, electrons from $v_{e}+n \rightarrow e^{-}+p$, and photons derived from events seen in the detector with electromagnetic showers pointing back to a disconnected veriex. Figures 2 and 3 illustrate the pulse height distributions for these samples as well as those of the signal plus background candidates (Fig. 2d, 3d). Over a wide range of pulse height cuts the size of the signal is quite insensitive. A typical choice is made such that $90 \%$ of the electrons are accepted as minimum ionizing.

Table II lists the data samples accumulated so far. The data presented here from our analysis to date, are containted in $I I(v)$ and onehalf of (II $(\bar{v})$ plus $\operatorname{III}(\bar{v})$.

In Fig. 4a are shown the $\theta^{2}$ distribution for the $\nu_{\mu}$ sample of those events classified as consistent with minimum ionization at the vertex. Figure $4 b$ are those identified as more than minimum ionizing. The distribution in $\theta^{2}$ of this latter sample is flat with no evidence of a peak at sma11 angles. A signal of $51 \pm 9$ electrons from reaction (1) is extracted from the $\theta^{2}$ distribution utilizing the region $\theta^{2}>0.01$ to establish the flat background level. Figure 5 a and 5 b show the pre1iminary $\theta^{2}$ distributions for a subset of the $\bar{v}_{\mu}$ data. A clear signal of $44 \pm 12$ events of reaction (2) is derived from this subset under the same procedure used for the $\nu_{\mu}$.

Our intended procedure for extracting \& final value of $\sin ^{2} \theta_{w}$ is to first normalize each reaction (1) and (2) separatily to the corresponding neutrino and anti-neutrino $f 1 u x$ by reference to the 10 - $Q^{2}$ quasi-elastic cross sections and then form the ratio, $R$.

Samples of reacions (4) and (5) are filtered from the raw data

$$
\begin{aligned}
& \nu_{\mu}+n+\mu^{-}+p \\
& \bar{\nu}_{\mu}+p \cdot \mu^{+}+n
\end{aligned}
$$


tapes simultaneously with those of reactions (1) and (2). Normalization cardidates are selected by requiring a single track at the vertex with an angle of $<15^{\circ}$ with respect to the beam axis and for which the single track has ange of at least 20 calorimeter wodules. Suttractions from the normalization sample must be made for inelastic backgrounds: reactions (6), (7), and (8) for $\nu_{\mu}$ and reactions (9), (10), and (11) for $\bar{v}_{\mu}$.

$$
\begin{aligned}
& \nu_{\mu}+p \rightarrow \mu^{-}+p+\pi^{+} \\
& \nu_{\mu}+n \rightarrow \mu^{-} p+\pi^{0} \\
& \nu_{\mu}+n \rightarrow \mu^{-} n+\pi^{+}
\end{aligned}
$$

$$
\begin{aligned}
& \bar{v}_{\mu}+\mathbf{n} \rightarrow \mu^{+}+\mathbf{n}+\pi^{-} \\
& \bar{v}_{\mu}+\mathbf{p} \rightarrow \mu^{+}+\mathbf{n}+\pi^{0} \\
& \bar{v}_{\mu}+\mathbf{p} \rightarrow \mu^{+}+\mathbf{p}+\pi^{-}
\end{aligned}
$$

Corrections must also be made to both signal and normalization channels for efficiencies and acceptance within cuts. This procedure hes been completed for the data $\operatorname{II}(v)$. For the sample $\operatorname{II}(\bar{v})$ and $\operatorname{III}(\bar{v})$, the analysis has recently started. Consequently, a result for only reaction (1) is presented together with preliminary progress on reaction (2).

Corrections to the reaction (1) signal of $51 \pm 9$ are made for losses (pulse height, 5\%; $\theta^{2}<0.01 \mathrm{rad}^{2}, 13 \% ; 210<\mathrm{E}<3200 \mathrm{MeV}, 24 \%$; angle reconstruction, scanning, and filtering, 21\%). Corrections to the reaction (4) normalization sample include background subtractions of $13 \%$ for reactions (6) p1us (8), $4 \%$ for reaction ( 7$), 16 \%$ for acceptance, and $24 \%$ for inefficiencies.

This analysis yields the result for reaction (1):

$$
\begin{gathered}
\sigma\left(v_{\mu}+\mathrm{e}^{-} \rightarrow v_{\mu}+\mathrm{e}^{-}\right)=[1.60 \pm 0.29(\text { stat. }) \pm 0.26(\text { sys. })] \times \mathrm{E}_{\nu}(\mathrm{GeV}) \times 10^{-42} \mathrm{~cm}^{2} \\
\text { Data analysis has recently begun for finding } \sigma\left(\bar{v}_{\mu}+\mathrm{e}^{-} \rightarrow \bar{v}_{\mu}+\mathrm{e}^{-}\right) .
\end{gathered}
$$
Studies have begun on internal consistency checks for the more difficult background ( 2 25\%) reactions (9), (10) and (11) which do not contain decaying $\pi^{+}$. Several corrections made to establish $\sigma\left(\nu_{\mu}+e^{-}+\nu_{\mu}+e^{-}\right)$ and $\sigma\left(\bar{v}_{\mu}+e^{-}+\bar{v}_{\mu}+e^{-}\right)$are common to both numerator and denominator in $R$; studies are underway to establish the level of compensation (or cancellation) thus provided and hence the limit of systematic error.

As can be seen from Table II and Figs. 4,5, we expect to obtain from our present data a total of approximately 150 events for reaction (1) and 90 events for reaction (2). Recently, the collaboration has been approved for additional extensive data-taking runs. With the presently increased average intensity of the AGS a doubling of the data sample is to be expected. Preliminary indications are that a final precision of $0.01-0.015$ on $\sin ^{2} \theta_{w}$ would then je possible from this experiment. 
This work is supported in part by the U. S. Department of Energy and the Japanese Mnistry of Education. Science and Culture through the Japan-USA Cooperative Research Project on High Energy Physics, and the US National Science Foundation.

\section{REFERENCES}

1. S. Sarantakos, A. Sirlin, W. J. Marciano, Nuclear Physics B217 (1983) 84 .

2. L. A. Ahrens et al., Physics Review Letters $\underline{51}$ (1983) 1514. 
BNL NEUTRINO DETECTOR

FRONT VIEW

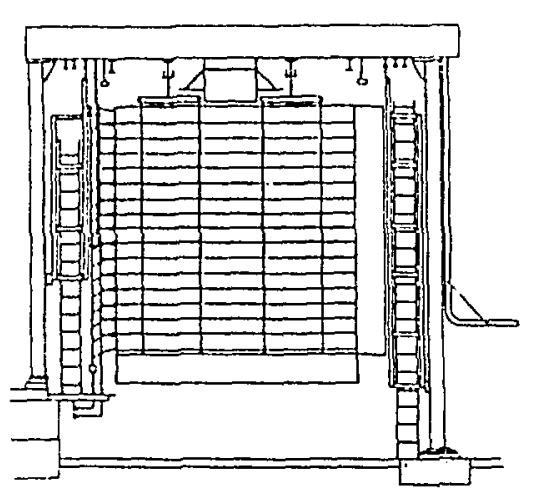

- HEUTRINO BEAM DIRECTION
DETAIL A

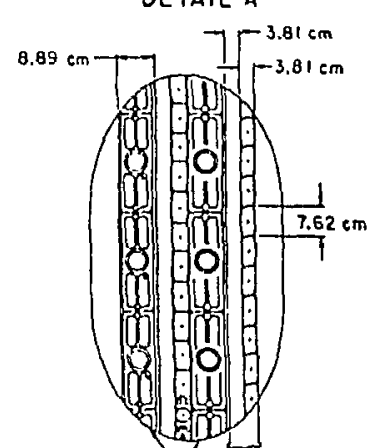

CALORIMETERS $D$ PROPORTIONAL

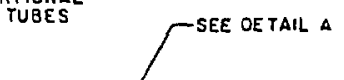

INSTITUTIONS

BNL

BROWN

OSAKA

TOKYO IINSI.

PENNSYLVANIA

SUNY STONT GROOK

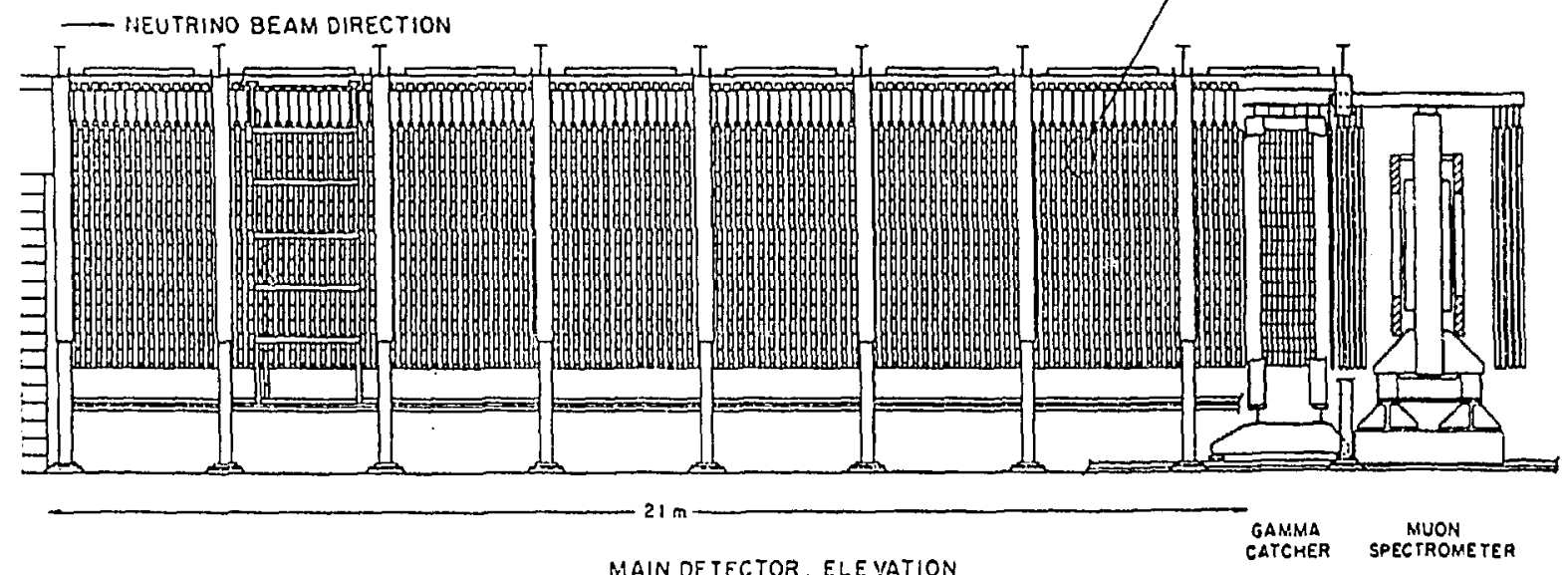

MAIN DETECTOR, ELE VATION

Figure 1. Elevation and detail of BNL/Brown/KEK/Osaka/Penasylvania/Stony Brook neutrino detector (E-734). 


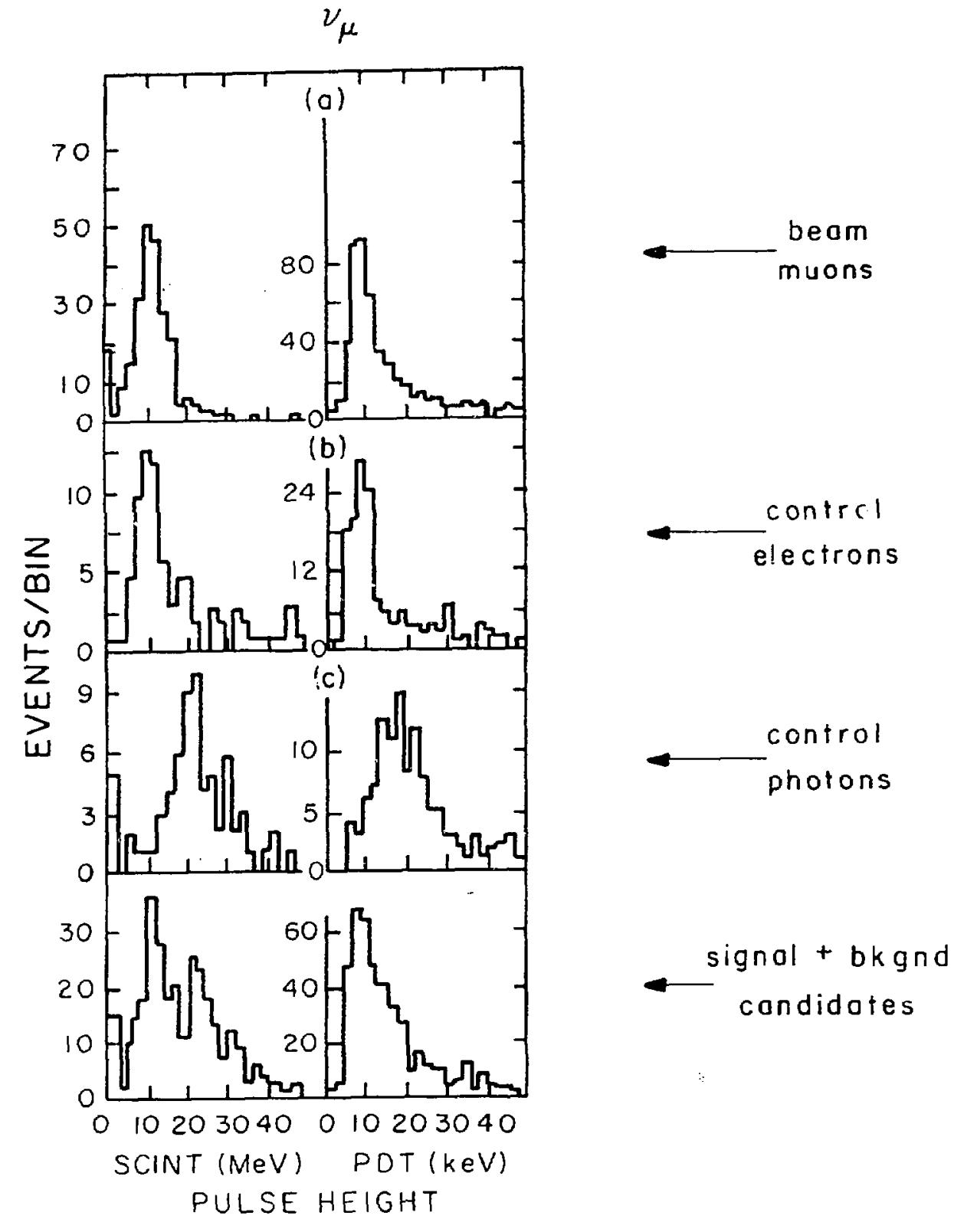

FIG. 2

Figure 2. Pulse height distributions for neutrinc data. Left column is for first calorimeter after vertex; right column is for $(X+Y)$ of first and second planes of proportional drift tubes.

(a) beam muons, (s) $+n e^{+}+$, (c) photon initiated. (d) signal plus background sample prior to separation by ionization. 


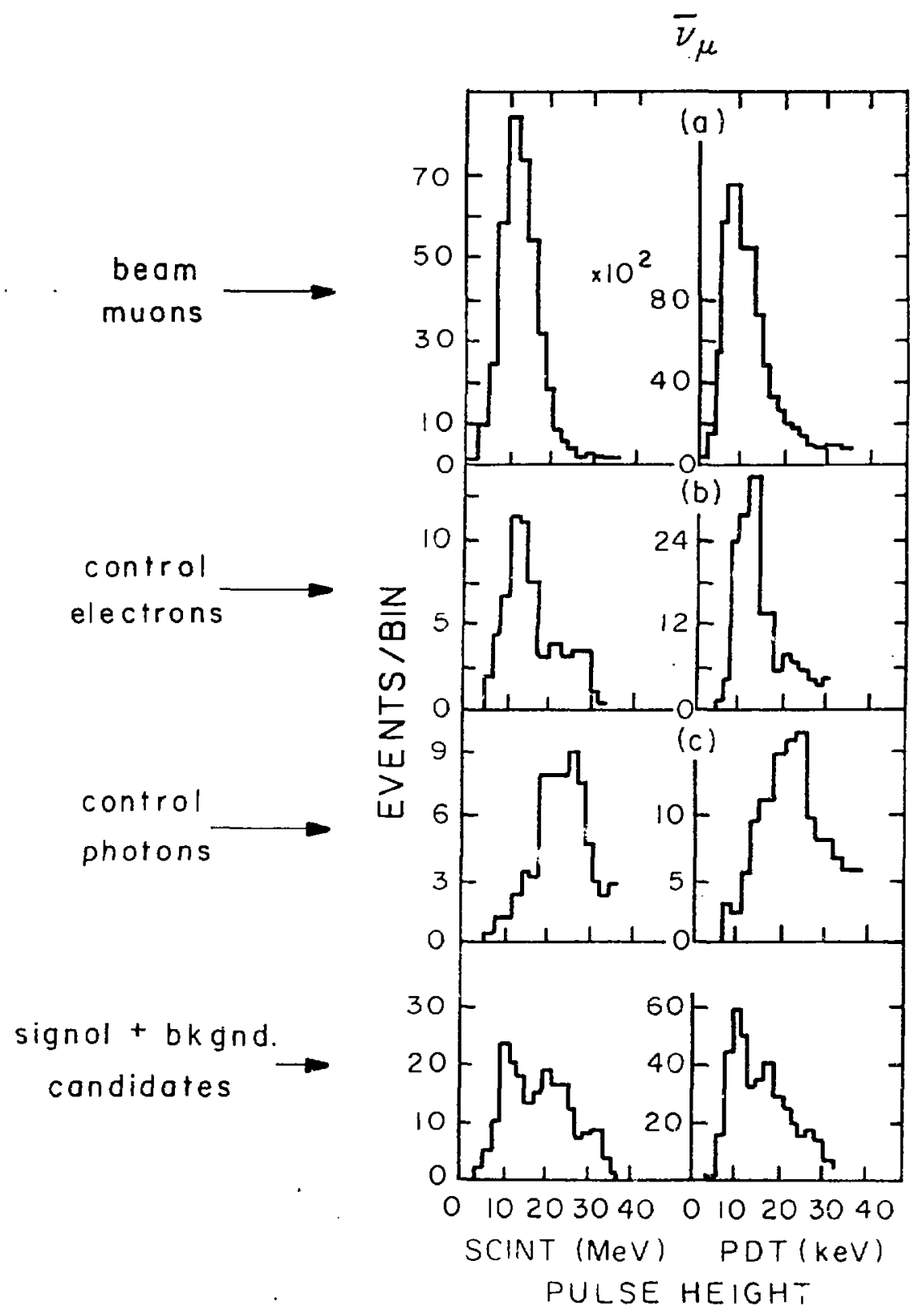

FIG 3

Figure 3. Same as Figure 2 but for anti-neutrino sample. 


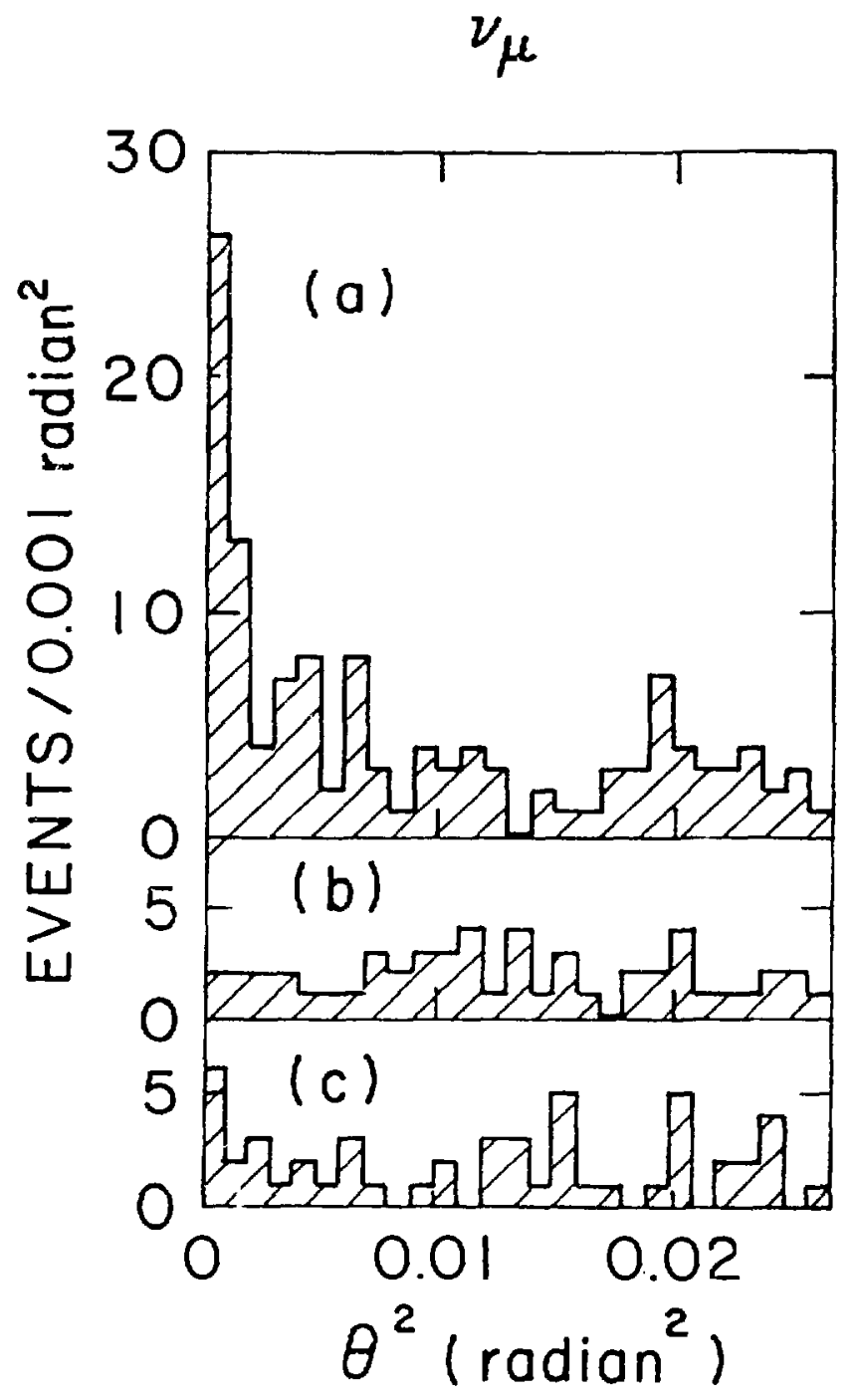

FIG. 4

Figure 4. $\theta^{2}$ distribution for neutrino data. (a) events satisfying minimum ionizing cut, (b) events failing minimum ionizing cut, (c) photon induced sample. 


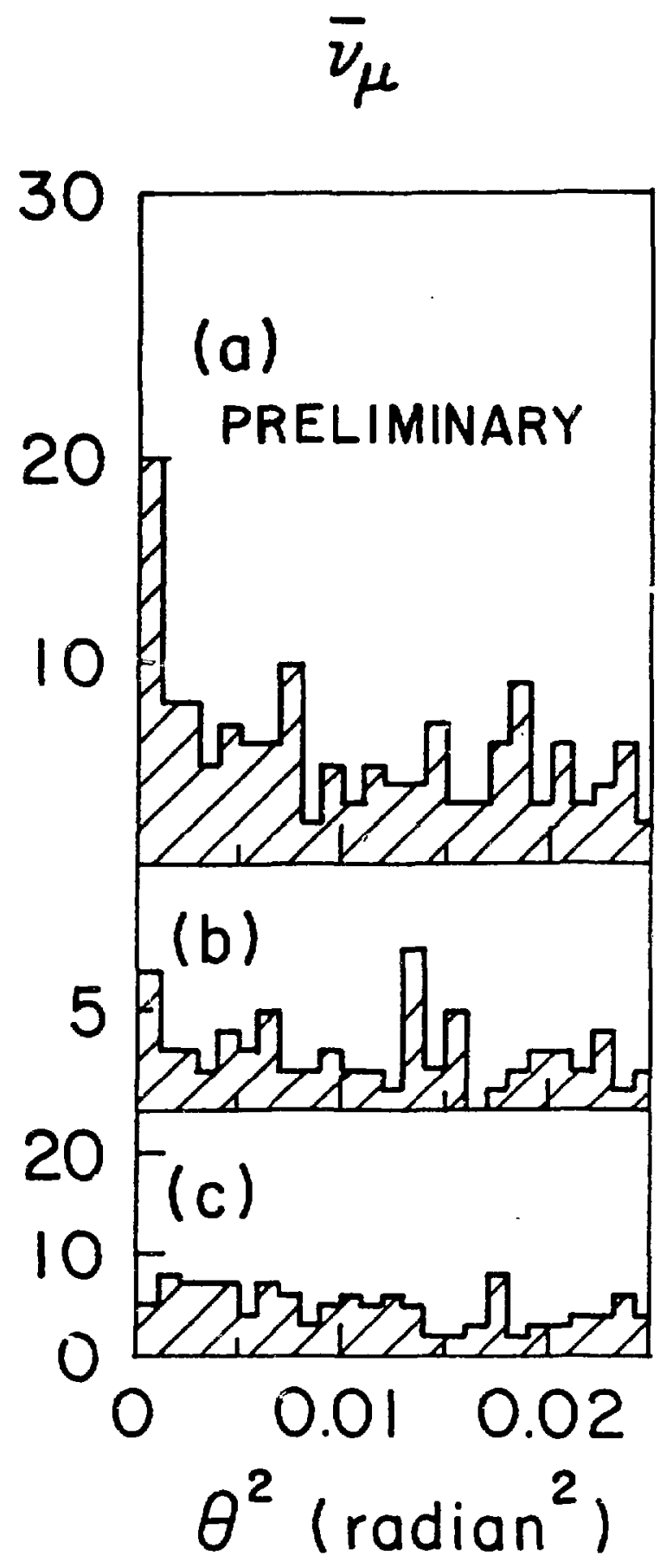

Figure 5. Same as Figure 4 but for antineutrino sample. 